\title{
Neural Network Model Reference Adaptive Control of Marine Vehicles
}

\author{
Alexander Leonessa, Tannen VanZwieten, and Yannick Morel \\ University of Central Florida \\ Department of Mechanical, Materials \& Aerospace Engineering \\ P.O. Box 162450, Orlando, FL 32816, USA \\ aleo@mail .ucf .edu, $\{$ tannen. vanzwieten, ymorel\}@gmail.com
}

Summary. A neural network model reference adaptive controller for trajectory tracking of nonlinear systems is developed. The proposed control algorithm uses a single layer neural network that bypasses the need for information about the system's dynamic structure and characteristics and provides portability. Numerical simulations are performed using nonlinear dynamic models of marine vehicles. Results are presented for two separate vehicle models, an autonomous surface vehicle and an autonomous underwater vehicle, to demonstrate the controller performance in terms of tuning, robustness, and tracking.

\section{Introduction}

Autonomous marine vehicles are used for a wide range of assignments, including oceanographic surveys, coastal patrols, pipeline maintenance, and mine hunting. Such missions require a high degree of agility and maneuverability, which can only be provided by a high-performance motion control system. We distinguish two generic types of marine vehicles: autonomous underwater vehicles (AUVs) and autonomous surface vehicles (ASVs). AUVs and ASVs are complementary, with each vehicle having distinct, mission specific advantages and disadvantages. This is enhanced by the prospect of collaborative control, where the ASVs can be used to transmit real time positioning and communication information to the AUVs through the air-sea interface.

The development of control algorithms for marine vehicles is the focus of several research groups around the world. For example, three different stabilization algorithms are developed in [6] for an underactuated hovercraft. However, the mathematical model for a hovercraft is simple when compared to that of a standard marine vehicle, as it does not contain many of the nonlinear, coupled drag terms that generally characterize marine vehicles. Furthermore, the tracking performance of the algorithm is not assessed, but instead the velocities are regulated to zero, which is a more fundamental problem. 
Many tracking controllers are available in the literature (see, for example, $[2,3,4,7,9,17,18])$. In [18], the case of a surface ship equipped with a pair of propellers is considered. This controller yields interesting results. However, the desired trajectory is limited to straight lines and circles. The same type of underactuation is considered in [17], where the authors derive a controller that uses a state estimator to handle state measurement uncertainties.

In [2] a global controller is designed that accounts for control amplitude saturation effects. Then, in [3], a velocity observer is added to the control algorithm, enabling the controller to work without velocity measurements.

A controller for an underactuated AUV equipped with a propeller and a side thruster is designed in [7]. The controller handles constant and slow varying external perturbations.

Typically, the design of a motion controller relies on the system's mathematical model. However, in the case of marine vehicles it is extremely challenging to obtain a model that will satisfactorily capture the dynamic behavior of the system. To compensate for this, one can use an adaptive approach to handle uncertainties in the geometric and and hydrodynamic constant parameters.

The design of adaptive controllers for marine vehicles has been widely studied. In [5], a nonlinear model-based adaptive output feedback controller was developed for a surface vessel. Global asymptotic position tracking was achieved assuming the structure of the mathematical model was of a particular form, with constant inertia and damping matrices. This structure was also extended to include a bias term representing drift, currents, and wave load. Simulations were presented, but did not demonstrate the controller robustness to unmodeled dynamics.

The dynamical behavior of a marine vehicle can only be partially depicted using current modeling techniques. These dynamics are especially challenging when the vehicle's velocity is not constant, as when it is following a search pattern or when its desired path is constantly being modified. Additionally, the ocean environment is characterized by large unknown perturbations. These features make it desirable to have a control system that is robust to model parameter and structure uncertainty.

This chapter introduces a neural network model reference adaptive controller (NN-MRAC) that has valuable self-tuning capabilities that allows it to adapt to the operating conditions in order to optimize the tracking performance of the closed loop system. The parameter update mechanism is derived using Lyapunov stability theory and guarantees that the tracking error is ultimately bounded when subject to some generalized constraints. The addition of a single layer neural network bypasses the need for information about the system's dynamic structure and characteristics. The control algorithm is applied to the surface vessel's nonlinear model presented in [19] and the AUV's nonlinear model from [14]. Numerical simulation results are presented for both cases. 


\section{Mathematical Preliminaries}

In this section we establish definitions, notation, and a key result used later in the chapter. Let $\mathbb{R}$ denote the set of real numbers, let $\mathbb{R}^{n}$ denote the set of $n \times 1$ real column vectors, let $\mathbb{R}^{n \times m}$ denote the set of real $n \times m$ matrices, and let $(\cdot)^{\mathrm{T}}$ denote transpose. Furthermore, $\|\cdot\|$ represents the Euclidean vector norm, and $A>0$ denotes the fact that the Hermitian matrix $A$ is positive definite. For a subset $\mathcal{S} \subset \mathbb{R}^{n}$, we write $\partial \mathcal{S}, \stackrel{\circ}{\mathcal{S}}$ for the boundary and the interior of $\mathcal{S}$, respectively.

In this chapter we consider nonlinear controlled dynamical systems of the form

$$
\dot{x}(t)=f(x(t), u(t)), \quad x(0)=x_{0}, \quad t \geq 0,
$$

where $x(t) \in \mathcal{D} \subseteq \mathbb{R}^{n}, t \geq 0$, is the system state vector, $\mathcal{D}$ is an open set, $0 \in \mathcal{D}, u(t) \in \mathcal{U} \subseteq \mathbb{R}^{m}, t \geq 0$, is the control input, $\mathcal{U}$ is the set of all admissible controls such that $u(\cdot)$ is a measurable function, and $f(\cdot, \cdot)$ is Lipschitz on $\mathcal{D} \times \mathcal{U}$. The closed-loop dynamical system corresponding to a feedback control $u(x(t), t), t \geq 0$, is given by

$$
\dot{x}(t)=\tilde{f}(x(t), t) \triangleq f(x(t), u(x(t), t)), \quad x(0)=x_{0}, \quad t \geq 0 .
$$

Following [12], the system (2) is said to be ultimately bounded if there is a compact set $M_{\mathrm{c}} \subset \mathcal{D}, 0 \in M_{\mathrm{c}}$, such that corresponding to each solution $x(t), t \geq 0$, of (2) there is a $T>0$ with the property that $x(t) \in M_{\mathrm{c}}$ for all $t>T$. The following theorem is introduced to establish a sufficient condition for ultimate boundedness.

Theorem 1. [12] Consider the closed loop nonlinear dynamical system (2) and assume that the forward solution $x(t), t \geq 0$, corresponding to an initial condition $x(0)=x_{0} \in \mathcal{D}$ exists. Assume that there exists a continuously differentiable function $V: \mathcal{D} \rightarrow \mathbb{R}$ and a compact set $M \subset D, 0 \in \stackrel{\circ}{M}$, such that

$$
\begin{aligned}
& V(0)=0, \quad V(x)>0, \quad x \in \mathcal{D} \backslash\{0\}, \\
& \frac{\mathrm{d} V(x)}{\mathrm{d} x} \tilde{f}(x, t) \leq-\epsilon<0, \quad x \in \mathcal{D} \backslash M, \quad t \geq 0,
\end{aligned}
$$

where $\epsilon>0$. Then (2) is ultimately bounded, that is, there exists a time $T>0$ and a compact set $M_{\mathrm{c}} \supseteq M$ such that $x(t) \in M_{\mathrm{c}}$ for all $t>T$.

Remark 1. From the proof of Theorem 1, it follows that the domain of convergence $M_{\mathrm{c}}$ is defined as the smallest level set associated with the Lyapunov function candidate $V(\cdot)$ that entirely contains the compact set $M$. 


\section{Modeling}

In this section we present a generic mathematical model of a marine vehicle that will be used for the development and testing of the controllers. In particular, our model assumes that pitch, roll and heave motions are negligible and feature only the three degrees of freedom corresponding to surge, sway, and yaw motions. Because of this choice we will assume that the vehicle's state space $\mathcal{D}$ coincides with $\mathbb{R}^{6}$, although the control algorithm can be easily extended to higher dimensions.

\subsection{Equations of Motion}

The notation used for the vehicle's generalized equations of motion follows [8], but is reduced to motion in the horizontal plane. The earth fixed frame (EFF), denoted by $x_{\mathrm{e}}, y_{\mathrm{e}}$ and $z_{\mathrm{e}}$, is chosen so that the vehicle's center of gravity is at the origin at time $t=0$. The $x_{\mathrm{e}}$ and $y_{\mathrm{e}}$ axes are directed toward the north and the east, respectively, while the $z_{\mathrm{e}}$ axis points downward in accordance with the right-hand rule. This frame is assumed to be inertial, the acceleration due to the earth's rotation being considered negligible. The vehicle's configuration in the EFF is

$$
\eta(t) \triangleq\left[x_{\mathrm{N}}(t), y_{\mathrm{E}}(t), \psi(t)\right]^{\mathrm{T}}, \quad t \geq 0
$$

where $x_{\mathrm{N}}(t) \in \mathbb{R}$ and $y_{\mathrm{E}}(t) \in \mathbb{R}$ describe the distance traveled along the $x_{\mathrm{e}}$ and $y_{\mathrm{e}}$ directions, respectively, and $\psi(t) \in \mathbb{R}$ describes the rotation about the $z_{\mathrm{e}}$ axis.

The body fixed frame (BFF) has its origin fixed at the vehicle's center of gravity, the $x_{\mathrm{b}}$ axis points forward, the $y_{\mathrm{b}}$ axis starboard, and the $z_{\mathrm{b}}$ axis downward. The vehicle's velocity is defined in the BFF as

$$
\nu(t) \triangleq[u(t), v(t), r(t)]^{\mathrm{T}}, \quad t \geq 0,
$$

where $u(t) \in \mathbb{R}$ and $v(t) \in \mathbb{R}$ are the components of the absolute velocity in the $x_{\mathrm{b}}$ and $y_{\mathrm{b}}$ directions, respectively, and $r(t) \in \mathbb{R}$ describes the angular velocity about the $z_{\mathrm{b}}$ axis. The vectors $\eta(t)$ and $\nu(t)$ are related by the kinematic equation [8],

$$
\dot{\eta}(t)=J(\eta(t)) \nu(t), \quad t \geq 0
$$

where

$$
J(\eta) \triangleq\left[\begin{array}{ccc}
\cos \psi & -\sin \psi & 0 \\
\sin \psi & \cos \psi & 0 \\
0 & 0 & 1
\end{array}\right],
$$

is the rotation matrix from the BFF to the EFF.

Using the form introduced in [8] and the previous notation, the marine vehicle's equation of motion is given by

$$
M \dot{\nu}(t)+C(\nu(t)) \nu(t)+D(\nu(t)) \nu(t)+g(\eta(t))=\hat{B} \tau(t), \quad t \geq 0,
$$


where $M \in \mathbb{R}^{3 \times 3}$ is the mass matrix (including added mass, see [19] for more details), $C(\nu(t)) \in \mathbb{R}^{3 \times 3}$ contains Coriolis, centripetal, and added-mass terms, $D(\nu(t)) \in \mathbb{R}^{3 \times 3}$ is the damping matrix, $g(\eta(t)) \in \mathbb{R}^{3}$ is the vector of restoring forces and moments, $\tau(t) \in \mathbb{R}^{m}$ is the input vector, and $\hat{B} \in \mathbb{R}^{3 \times m}$ characterizes how the control inputs affect the dynamics of the vehicle.

While the rigid body inertia, Coriolis, centripetal, and gravitational terms are described in [8], the hydrodynamic terms are much more challenging to model and depend on the particular geometry of the considered vehicle. In general, even very thorough hydrodynamic modeling efforts are only able to partially describe the dynamic behavior of a marine vehicle, as the assumptions made always considerably affect the final result. In light of these considerations we are going to write the vehicle dynamics as

$$
\dot{\nu}(t)=f(x(t))+B \tau(t), \quad t \geq 0,
$$

where $x \triangleq\left[\eta^{\mathrm{T}} \nu^{\mathrm{T}}\right]^{\mathrm{T}}$ is the state vector and

$$
\begin{aligned}
B & \triangleq M^{-1} \hat{B}, \\
f(x) & \triangleq-M^{-1}[C(\nu)+D(\nu)] \nu-M^{-1} g(\eta),
\end{aligned}
$$

are assumed to be unknown.

\section{Adaptive Controller Design}

The nonlinearities, unmodeled dynamics, and strong dynamic coupling inherent to marine vehicle models make it desirable to have a multi-input/multioutput control system that is capable of self-tuning. We chose to solve this problem using an adaptive control approach. The stability analysis and the parameter update mechanism are derived using Lyapunov stability theory.

To simplify the presentation, the explicit dependence of the state variables upon time, when obvious, will be omitted.

\subsection{Reference System}

When using model reference adaptive control, a control algorithm is developed so that the system mimics the behavior of a reference system that provides smooth convergence to the desired trajectory. Choosing the appropriate reference system allows the vehicle to exhibit less overshoot and oscillatory behavior as well as better tracking performance. Furthermore, the control inputs become more realistic, even when the vehicle is far away from the desired trajectory, diminishing the need to implement input amplitude and rate saturation algorithms.

We consider a linear reference system that can be written as

$$
\dot{x}_{\mathrm{r}}(t)=A_{\mathrm{r}} x_{\mathrm{r}}(t)+B_{\mathrm{r}} \hat{r}(t), \quad t \geq 0,
$$


where $x_{\mathrm{r}}(t) \in \mathbb{R}^{2 m}$ is the reference state, $A_{\mathrm{r}} \in \mathbb{R}^{2 m \times 2 m}, B_{\mathrm{r}} \in \mathbb{R}^{2 m \times m}$ are constant matrices, and $\hat{r}(t) \in \mathbb{R}^{m}$ is the reference input.

The reference system considered here is composed of three uncoupled second-order oscillators. Each oscillator is characterized by a damping coefficient $\zeta_{i}>0, i=1, \ldots, m$ and a natural frequency $w_{0 i}>0, i=1, \ldots, m$. This choice was mostly motivated by the simplicity of the corresponding reference dynamics. The dynamics of the $i^{\text {th }}$ oscillator are given by

$$
\ddot{x}_{\mathrm{r} i}(t)+2 \zeta_{i} \omega_{0 i} \dot{x}_{\mathrm{r} i}(t)+\omega_{0 i}^{2} x_{\mathrm{r} i}(t)=\omega_{0 i}^{2} \hat{r}_{i}(t), \quad t \geq 0, \quad i=1, \ldots, m .
$$

The reference system can thus be rewritten as

$$
\left[\begin{array}{l}
\dot{x}_{1 \mathrm{r}}(t) \\
\dot{x}_{2 \mathrm{r}}(t)
\end{array}\right]=\left[\begin{array}{cc}
0_{m} & I_{m} \\
-\omega_{0}^{2} & -A_{\mathrm{rm}}
\end{array}\right]\left[\begin{array}{l}
x_{1 \mathrm{r}}(t) \\
x_{2 \mathrm{r}}(t)
\end{array}\right]+\left[\begin{array}{c}
0_{m} \\
\omega_{0}^{2}
\end{array}\right] \hat{r}(t), \quad t \geq 0
$$

where

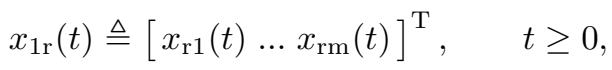

$$
\begin{aligned}
& x_{2 \mathrm{r}}(t) \triangleq\left[\dot{x}_{\mathrm{r} 1}(t) \ldots \dot{x}_{\mathrm{rm}}(t)\right]^{\mathrm{T}}, \quad t \geq 0,
\end{aligned}
$$

and

$$
\begin{aligned}
A_{\mathrm{rm}} & \triangleq \operatorname{diag}\left(2 \zeta_{1} \omega_{01}, \ldots, 2 \zeta_{p} \omega_{0 m}\right), \\
\omega_{0} & \triangleq \operatorname{diag}\left(\omega_{01}, \ldots, \omega_{0 m}\right) .
\end{aligned}
$$

Finally, the desired trajectory for the marine vehicle may be written as

$$
\tilde{x}_{\mathrm{d}}(t)=\left[x_{\mathrm{d} 1}(t) \ldots x_{\mathrm{d} m}(t)\right]^{\mathrm{T}}, \quad t \geq 0 .
$$

By choosing

$$
\hat{r}(t)=\omega_{0}^{-2}\left(\ddot{\tilde{x}}_{\mathrm{d}}(t)+A_{\mathrm{rm}} \dot{\tilde{x}}_{\mathrm{d}}(t)+\omega_{0}^{2} \tilde{x}_{\mathrm{d}}(t)\right), \quad t \geq 0,
$$

we find that

$$
\left[\begin{array}{l}
\dot{x}_{1 \mathrm{r}}(t)-\dot{\tilde{x}}_{\mathrm{d}}(t) \\
\dot{x}_{2 \mathrm{r}}(t)-\ddot{\tilde{x}}_{\mathrm{d}}(t)
\end{array}\right]=A_{\mathrm{r}}\left[\begin{array}{l}
x_{1 \mathrm{r}}(t)-\tilde{x}_{\mathrm{d}}(t) \\
x_{2 \mathrm{r}}(t)-\dot{\tilde{x}}_{\mathrm{d}}(t)
\end{array}\right], \quad t \geq 0
$$

Since

$$
A_{\mathrm{r}} \triangleq\left[\begin{array}{cc}
0_{m} & I_{m} \\
-\omega_{0}^{2} & -A_{\mathrm{rm}}
\end{array}\right]
$$

is Hurwitz, it follows that $x_{1 \mathrm{r}}(t)-\tilde{x}_{\mathrm{d}}(t) \rightarrow 0$ and $x_{2 \mathrm{r}}(t)-\dot{\tilde{x}}_{\mathrm{d}}(t) \rightarrow 0$ as $t \rightarrow \infty$, i.e., the reference state converges to the desired trajectory. The remaining problem is to design a control command, $\tau(t) \in \mathbb{R}^{m}$, such that the tracking error converges to a fixed neighborhood around the origin. Considering that the mass, Coriolis/centrifugal, and damping matrices of the real system contain unknown parameters and unknown terms, a control signal that accounts for these uncertainties needs to be considered. 


\subsection{Control Command}

The next goal is to define a control signal, $\tau \in \mathbb{R}^{m}$, that guarantees that the tracking error is ultimately bounded.

Theorem 2. Consider the vehicle dynamics (7), (10) and the reference dynamics (13). Introduce a tracking error $e_{1}\left(\eta, x_{1 \mathrm{r}}\right) \in \mathbb{R}^{m}$, where $\eta \in \mathbb{R}^{n}$ and $x_{1 \mathrm{r}} \in \mathbb{R}^{m}$ represent the vehicle and reference configuration, respectively, such that $e_{1}\left(\eta(t), x_{1 \mathrm{r}}(t)\right) \equiv 0, t \geq 0$, if and only if perfect tracking is achieved. Assume that the error dynamics can be written in the form

$$
\dot{e}_{1}(t)=Q_{1}\left(\eta(t), x_{1 \mathrm{r}}(t)\right) q_{2}\left(x(t), x_{\mathrm{r}}(t), \chi(t)\right), \quad t \geq 0,
$$

where $\chi(t) \in \mathbb{R}^{m}$ is an exogenous signal, $x(t) \in \mathcal{D}$ is the state of the system, $x_{\mathrm{r}}(t) \in \mathbb{R}^{2 m}$ is the reference state, $Q_{1}: \mathbb{R}^{n} \times \mathbb{R}^{m} \rightarrow \mathbb{R}^{m \times m}$, and $q_{2}: \mathbb{R}^{2 n} \times$ $\mathbb{R}^{2 m} \times \mathbb{R}^{m} \rightarrow \mathbb{R}^{m}$. Assume also that there exists a Lyapunov function candidate $V_{s}\left(e_{1}\right)$ such that $\frac{\mathrm{d} V_{s}\left(e_{1}\right)}{\mathrm{d} e_{1}}=0$ if and only if $e_{1}=0$. Next, consider a control command

$$
\tau^{*}\left(x, x_{\mathrm{r}}, \chi, \hat{r}\right)=-\Lambda_{1}\left[\Theta^{*} w\left(x, x_{\mathrm{r}}, \chi, \hat{r}\right)+\delta^{*}(x)\right],
$$

where $\tau^{*}\left(x, x_{\mathrm{r}}, \chi, \hat{r}\right) \in \mathbb{R}^{m}, \Lambda_{1} \in \mathbb{R}^{m \times n}$ is such that $B \Lambda_{1}$ is nonsingular, $\Theta^{*} \in \mathbb{R}^{n \times m}, w\left(x, x_{\mathrm{r}}, \chi, \hat{r}\right) \in \mathbb{R}^{m}$, and $\delta^{*}(x) \in \mathbb{R}^{n}$. Furthermore, let

$$
\begin{aligned}
\Theta^{*}= & \left(B \Lambda_{1}\right)^{-1} \Lambda_{2} \\
w\left(x, x_{r}, \chi, \hat{r}\right)= & \left(\frac{\partial q_{2}\left(x, x_{\mathrm{r}}, \chi\right)}{\partial \nu} \Lambda_{2}\right)^{-1}\left(\frac{\partial q_{2}\left(x, x_{\mathrm{r}}, \chi\right)}{\partial \eta} J(\eta) \nu+\frac{\partial q_{2}\left(x, x_{\mathrm{r}}, \chi\right)}{\partial x_{\mathrm{r}}} \dot{x}_{\mathrm{r}}\right. \\
& +\frac{\partial q_{2}\left(x, x_{\mathrm{r}}, \chi\right)}{\partial \chi} \dot{\chi}-\dot{q}_{2 \mathrm{des}}\left(x, x_{\mathrm{r}}\right)+e_{2}\left(x, x_{\mathrm{r}}, \chi\right) \\
& \left.+G_{2}^{-1} Q_{1}^{\mathrm{T}}\left(\eta, x_{1 \mathrm{r}}\right) \frac{\mathrm{d} V_{s}\left(e_{1}\right)^{\mathrm{T}}}{\mathrm{d} e_{1}}\right) \\
\delta^{*}(x)= & \left(B \Lambda_{1}\right)^{-1} f(x)
\end{aligned}
$$

with

$$
\begin{aligned}
q_{2 \mathrm{des}}\left(\eta, x_{1 \mathrm{r}}\right) & =-\alpha\left(\eta, x_{1 \mathrm{r}}\right) G_{1} Q_{1}^{\mathrm{T}}\left(\eta, x_{1 \mathrm{r}}\right){\frac{\mathrm{d} V_{s}\left(e_{1}\right)^{\mathrm{T}}}{\mathrm{d} e_{1}}}_{e_{2}\left(x, x_{\mathrm{r}}, \chi\right)}=q_{2}\left(x, x_{\mathrm{r}}, \chi\right)-q_{2 \mathrm{des}}\left(\eta, x_{1 \mathrm{r}}\right)
\end{aligned}
$$

where $\alpha: \mathbb{R}^{n} \times \mathbb{R}^{m} \rightarrow \mathbb{R}^{+}, G_{1} \in \mathbb{R}^{m \times m}$ is positive definite, and $\Lambda_{2} \in \mathbb{R}^{n \times m}$ is such that $\frac{\partial q_{2}\left(x, x_{\mathrm{r}}, \chi\right)}{\partial \nu} \Lambda_{2}$ is nonsingular. Then the error dynamics associated with the closed loop given by (7), (10), (13), and (23) are ultimately bounded.

Proof. Using (22), the derivative of the Lyapunov function candidate $V_{s}\left(e_{1}\right)$ is given by 


$$
\dot{V}_{s}\left(x, x_{\mathrm{r}}, \chi\right)=\frac{\mathrm{d} V_{s}\left(e_{1}\right)}{\mathrm{d} e_{1}} Q_{1}\left(\eta, x_{1 \mathrm{r}}\right) q_{2}\left(x, x_{\mathrm{r}}, \chi\right) .
$$

Using a backstepping approach derived from [11], we will use $q_{2}\left(x, x_{\mathrm{r}}, \chi\right)$ as a virtual control command. Ideally $q_{2}\left(x, x_{\mathrm{r}}, \chi\right)$ would be equal to $q_{2 \mathrm{des}}\left(\eta, x_{1 \mathrm{r}}\right)$ defined by (27), such that

$$
\left.\dot{V}_{s}\left(x, x_{\mathrm{r}}, \chi\right)\right|_{q_{2}=q_{2 \mathrm{des}}}=-\alpha\left(\eta, x_{1 \mathrm{r}}\right) \frac{\mathrm{d} V_{s}\left(e_{1}\right)}{\mathrm{d} e_{1}} Q_{1}\left(\eta, x_{1 \mathrm{r}}\right) G_{1} Q_{1}^{\mathrm{T}}\left(\eta, x_{1 \mathrm{r}}\right){\frac{\mathrm{d} V_{s}\left(e_{1}\right)^{\mathrm{T}}}{\mathrm{d} e_{1}}}^{\mathrm{T}}
$$

which is negative definite. Next, consider a new Lyapunov function candidate,

$$
V^{*}\left(e_{1}, e_{2}\right)=V_{s}\left(e_{1}\right)+\frac{1}{2} e_{2}^{\mathrm{T}} G_{2} e_{2}
$$

where $G_{2} \in \mathbb{R}^{m \times m}$ is positive definite and $e_{2}$ is defined by (28). The time derivative of $(31)$ is of the form

$$
\dot{V}^{*}\left(x, x_{\mathrm{r}}, \chi, \hat{r}, \tau^{*}\right)=\dot{V}_{s}\left(e_{1}\right)+e_{2}^{\mathrm{T}}\left(x, x_{\mathrm{r}}, \chi\right) G_{2} \dot{e}_{2}\left(x, x_{\mathrm{r}}, \chi, \hat{r}, \tau^{*}\right) .
$$

Next, taking the derivative of (28) and substituting the kinematic and dynamic equations (7) and (10), we find the error dynamics to be

$$
\begin{aligned}
\dot{e}_{2}\left(x, x_{\mathrm{r}}, \chi, \hat{r}, \tau^{*}\right)= & \frac{\partial q_{2}\left(x, x_{\mathrm{r}}, \chi\right)}{\partial \eta} J(\eta) \nu+\frac{\partial q_{2}\left(x, x_{\mathrm{r}}, \chi\right)}{\partial \nu}\left(f(x)+B \tau^{*}\right) \\
& +\frac{\partial q_{2}\left(x, x_{\mathrm{r}}, \chi\right)}{\partial x_{\mathrm{r}}} \dot{x}_{\mathrm{r}}+\frac{\partial q_{2}\left(x, x_{\mathrm{r}}, \chi\right)}{\partial \chi} \dot{\chi}-\dot{q}_{2 \mathrm{des}}\left(x, x_{\mathrm{r}}\right),
\end{aligned}
$$

which, after substituting the control input (23), provides the following closedloop error dynamics

$$
\begin{aligned}
& \dot{e}_{1}\left(x, x_{\mathrm{r}}, \chi\right)=Q_{1}\left(\eta, x_{1 \mathrm{r}}\right) q_{2}\left(x, x_{\mathrm{r}}, \chi\right), \\
& \dot{e}_{2}\left(x, x_{\mathrm{r}}, \chi\right)=-e_{2}\left(x, x_{\mathrm{r}}, \chi\right)-G_{2}^{-1} Q_{1}^{\mathrm{T}}\left(\eta, x_{1 \mathrm{r}}\right){\frac{\mathrm{d} V_{s}\left(e_{1}\right)^{\mathrm{T}}}{\mathrm{d} e_{1}} .}^{\mathrm{T}} .
\end{aligned}
$$

Therefore, (32) becomes

$$
\begin{aligned}
\dot{V}^{*}\left(x, x_{\mathrm{r}}, \chi\right)= & -\alpha\left(\eta, x_{1 \mathrm{r}}\right) \frac{\mathrm{d} V_{s}\left(e_{1}\right)}{\mathrm{d} e_{1}} Q_{1}\left(\eta, x_{1 \mathrm{r}}\right) G_{1} Q_{1}^{\mathrm{T}}\left(\eta, x_{1 \mathrm{r}}\right) \frac{\mathrm{d} V_{s}\left(e_{1}\right)^{\mathrm{T}}}{\mathrm{d} e_{1}} \\
& -e_{2}^{\mathrm{T}}\left(x, x_{\mathrm{r}}, \chi\right) G_{2} e_{2}\left(x, x_{\mathrm{r}}, \chi\right)
\end{aligned}
$$

which is negative definite, proving asymptotic stability of the closed-loop error dynamics. This concludes our proof.

Remark 2. The matrix $\Theta^{*}$ and the function $\delta^{*}(x)$ in (23) are unknown, while $w\left(x, x_{\mathrm{r}}, \chi, \hat{r}\right)$ is a known function of the states and the reference input. 
Remark 3. The particular choice for the error dynamics (22) is motivated by the individual application of Theorem 2 to marine vehicles. Such a statement is not limiting in any way since we can always choose $Q_{1}\left(\eta, x_{1 \mathrm{r}}\right)=I_{m}$ and $\chi(t) \equiv 0$ for all $t \geq 0$.

Theorem 3. Consider the system, tracking error, and virtual command described in Theorem 2, with the additional condition that $\frac{\partial q_{2}\left(x, x_{\mathrm{r}}, \chi\right)}{\partial \nu}$ is bounded on $\mathbb{R}^{2 n} \times \mathbb{R}^{2 m} \times \mathbb{R}^{m}$ and $B \Lambda_{1}$ is positive definite. Let the controller (23) be replaced with the following:

$$
\tau(t)=-\Lambda_{1}\left[\Theta(t) w\left(x(t), x_{\mathrm{r}}(t), \chi(t), \hat{r}(t)\right)+W(t) \sigma(x(t))\right], \quad t \geq 0,
$$

where $\Theta(t) \in \mathbb{R}^{n \times m}$ and $W(t) \in \mathbb{R}^{n \times q}, t \geq 0$, are parameter estimates, and $\sigma(x) \in \mathbb{R}^{q}, x \in \mathbb{R}^{n}$, is a vector composed of basis functions that we use to approximate the system's dynamics with a maximum approximation error $\varepsilon^{*}>0$. Furthermore, let the parameter update laws be

$$
\begin{aligned}
\dot{\Theta} & =\frac{\partial q_{2}\left(x, x_{\mathrm{r}}, \chi\right)^{\mathrm{T}}}{\partial \nu} G_{2}^{\mathrm{T}} e_{2}\left(x, x_{\mathrm{r}}, \chi\right) w^{\mathrm{T}}\left(x, x_{\mathrm{r}}, \chi, \hat{r}\right) \Gamma_{1}-\sigma_{1} \Theta, \\
\dot{W} & =\frac{\partial q_{2}\left(x, x_{\mathrm{r}}, \chi\right)^{\mathrm{T}}}{\partial \nu} G_{2}^{\mathrm{T}} e_{2}\left(x, x_{\mathrm{r}}, \chi\right) \sigma^{\mathrm{T}}(x) \Gamma_{2}-\sigma_{2} W,
\end{aligned}
$$

where $\Gamma_{1} \in \mathbb{R}^{m \times m}$ and $\Gamma_{2} \in \mathbb{R}^{q \times q}$ are positive definite $\sigma_{1} \geq 0$, and $\sigma_{2} \geq 0$. Then the tracking error and the parameter estimates are ultimately bounded with a domain of convergence defined as

$$
M_{\mathrm{c}} \triangleq\left\{\left(e_{1}, e_{2}, \tilde{\Theta}, \tilde{W}\right): V\left(e_{1}, e_{2}, \tilde{\Theta}, \tilde{W}\right) \leq \alpha\right\}
$$

where

$V\left(e_{1}, e_{2}, \tilde{\Theta}, \tilde{W}\right)=V_{s}\left(e_{1}\right)+\frac{1}{2} e_{2}^{\mathrm{T}} G_{2} e_{2}+\frac{1}{2} \operatorname{tr}\left(B \Lambda_{1} \tilde{\Theta} \Gamma_{1}^{-1} \tilde{\Theta}^{\mathrm{T}}\right)+\frac{1}{2} \operatorname{tr}\left(B \Lambda_{1} \tilde{W} \Gamma_{2}^{-1} \tilde{W}^{\mathrm{T}}\right)$,

and

$$
\begin{gathered}
\alpha \triangleq \max _{\left(e_{1}, e_{2}, \tilde{\Theta}, \tilde{W}\right) \in M} V\left(e_{1}, e_{2}, \tilde{\Theta}, \tilde{W}\right) \\
M \triangleq\left\{\left(e_{1}, e_{2}, \tilde{\Theta}, \tilde{W}\right):\left\|G_{2}^{\frac{1}{2}} e_{2}\left(x, x_{\mathrm{r}}, \chi\right)\right\| \leq\left\|G_{2}^{\frac{1}{2}} \frac{\partial q_{2}\left(x, x_{\mathrm{r}}, \chi\right)}{\partial \nu} B \Lambda_{1}\right\| \varepsilon^{*},\right. \\
\operatorname{tr}\left(B \Lambda_{1} \tilde{\Theta} \Gamma_{1}^{-1} \tilde{\Theta}^{\mathrm{T}}\right) \leq \operatorname{tr}\left(B \Lambda_{1} \Theta^{*} \Gamma_{1}^{-1} \Theta^{* \mathrm{~T}}\right), \\
\left.\operatorname{tr}\left(B \Lambda_{1} \tilde{W} \Gamma_{2}^{-1} \tilde{W}^{\mathrm{T}}\right) \leq \operatorname{tr}\left(B \Lambda_{1} W^{*} \Gamma_{2}^{-1} W^{* \mathrm{~T}}\right)\right\} .
\end{gathered}
$$

Proof. Since $\Theta^{*}$ and $\delta^{*}(x)$ from Theorem 2 are unknown, their estimates need to be introduced. In particular, $\Theta^{*}$ in $(23)$ will be replaced with its estimate 
$\Theta(t)$, such that $\Theta(t)=\Theta^{*}+\tilde{\Theta}(t)$, where $\tilde{\Theta}(t)$ represents the estimation error. Following the approach described in [10], it will be assumed that the vector function $\delta^{*}(x)$ can be approximated by a linear parameterized neural network with a maximum approximation error given by $\varepsilon^{*}>0$. Hence, there exists $\varepsilon(x)$ such that $\|\varepsilon(x)\|<\varepsilon^{*}$ for all $x \in \mathbb{R}^{2 n}$, and

$$
\delta^{*}(x) \triangleq W^{*} \sigma(x)+\varepsilon(x), \quad x \in \mathbb{R}^{2 n},
$$

where $W^{*} \in \mathbb{R}^{m \times q}$ is the matrix of unknown control gain weights (constant) that minimize the approximation error, $\sigma: \mathbb{R}^{2 n} \rightarrow \mathbb{R}^{q}$ is a vector of basis functions such that each component of $\sigma(\cdot)$ takes values in $[0,1], \varepsilon(\cdot)$ is the vector of approximation errors, and $\left\|W^{*}\right\| \leq w^{*}$, where $w^{*}$ is a bound for the unknown control gain optimal weight matrix. Next, following the procedure described in [13], $\delta^{*}(x(t))$ is replaced in (23) with $W(t) \sigma(x(t))$ where $W(t) \in$ $\mathbb{R}^{m \times q}$ is the estimate of the weights such that $W(t)=W^{*}+\tilde{W}(t)$, with $\tilde{W}(t)$ representing the estimation error.

When replacing the control command (23) with (37), the corresponding closed-loop error dynamics are given by

$$
\begin{aligned}
\dot{e}_{1}\left(x, x_{\mathrm{r}}, \chi\right)= & Q_{1}\left(\eta, x_{1 \mathrm{r}}\right) q_{2}\left(x, x_{\mathrm{r}}, \chi\right), \\
\dot{e}_{2}\left(x, x_{\mathrm{r}}, \chi, \tilde{\Theta}, \tilde{W}, \hat{r}\right)= & -e_{2}\left(x, x_{\mathrm{r}}, \chi\right)-G_{2}^{-1} Q_{1}^{\mathrm{T}}\left(\eta, x_{1 \mathrm{r}}\right){\frac{\mathrm{d} V_{s}\left(e_{1}\right)}{\mathrm{d} e_{1}}}^{\mathrm{T}} \\
& +\frac{\partial q_{2}\left(x, x_{\mathrm{r}}, \chi\right)}{\partial \nu} \gamma\left(x, x_{\mathrm{r}}, \chi, \tilde{\Theta}, \tilde{W}, \hat{r}\right),
\end{aligned}
$$

where

$$
\gamma\left(x, x_{\mathrm{r}}, \chi, \tilde{\Theta}, \tilde{W}, \hat{r}\right) \triangleq-B \Lambda_{1}\left[\tilde{\Theta} w\left(x, x_{\mathrm{r}}, \chi, \hat{r}\right)+\tilde{W} \sigma(x)-\varepsilon(x)\right] .
$$

To show ultimate boundedness of the closed-loop error dynamics given by (45) and (46), the following Lyapunov function candidate is considered:

$$
V\left(e_{1}, e_{2}, \tilde{\Theta}, \tilde{W}\right)=V^{*}\left(e_{1}, e_{2}\right)+\frac{1}{2} \operatorname{tr}\left(B \Lambda_{1} \tilde{\Theta} \Gamma_{1}^{-1} \tilde{\Theta}^{\mathrm{T}}\right)+\frac{1}{2} \operatorname{tr}\left(B \Lambda_{1} \tilde{W} \Gamma_{2}^{-1} \tilde{W}^{\mathrm{T}}\right) .
$$

Note that $V\left(e_{1}, e_{2}, \tilde{\Theta}, \tilde{W}\right)$ is a positive definite scalar function with continuous partial derivatives, in accordance with the hypothesis set by Theorem 1 . The corresponding Lyapunov derivative is given by

$$
\begin{aligned}
& \dot{V}\left(x, x_{\mathrm{r}}, \chi, \tilde{\Theta}, \tilde{W}, \hat{r}\right)=\frac{\partial V^{*}\left(e_{1}, e_{2}\right)}{\partial e_{1}} \dot{e}_{1}\left(x, x_{\mathrm{r}}, \chi\right)+\frac{\partial V^{*}\left(e_{1}, e_{2}\right)}{\partial e_{2}} \dot{e}_{2}\left(x, x_{\mathrm{r}}, \chi, \tilde{\Theta}, \tilde{W}, \hat{r}\right) \\
& +\operatorname{tr}\left(B \Lambda_{1} \tilde{\Theta} \Gamma_{1}^{-1} \dot{\Theta}^{\mathrm{T}}\right)+\operatorname{tr}\left(B \Lambda_{1} \tilde{W} \Gamma_{2}^{-1} \dot{W}^{\mathrm{T}}\right) \\
& =-\alpha\left(\eta, x_{1 \mathrm{r}}\right) \frac{\mathrm{d} V_{s}\left(e_{1}\right)}{\mathrm{d} e_{1}} Q_{1}\left(\eta, x_{1 \mathrm{r}}\right) G_{1} Q_{1}^{\mathrm{T}}\left(\eta, x_{1 \mathrm{r}}\right){\frac{\mathrm{d} V_{s}\left(e_{1}\right)^{\mathrm{T}}}{\mathrm{d} e_{1}}}^{\mathrm{T}} \\
& -e_{2}^{\mathrm{T}}\left(x, x_{\mathrm{r}}, \chi\right) G_{2} e_{2}\left(x, x_{\mathrm{r}}, \chi\right)+e_{2}^{\mathrm{T}}\left(x, x_{\mathrm{r}}, \chi\right) G_{2} \frac{\partial q_{2}\left(x, x_{\mathrm{r}}, \chi\right)}{\partial \nu} B \Lambda_{1} \varepsilon(x)
\end{aligned}
$$




$$
\begin{aligned}
& -\operatorname{tr}\left(B \Lambda_{1} \tilde{\Theta} w\left(x, x_{\mathrm{r}}, \chi, \hat{r}\right) e_{2}^{\mathrm{T}}\left(x, x_{\mathrm{r}}, \chi\right) G_{2} \frac{\partial q_{2}\left(x, x_{\mathrm{r}}, \chi\right)}{\partial \nu}-B \Lambda_{1} \tilde{\Theta} \Gamma_{1}^{-1} \dot{\Theta}^{\mathrm{T}}\right) \\
& -\operatorname{tr}\left(B \Lambda_{1} \tilde{W} \sigma(x) e_{2}^{\mathrm{T}}\left(x, x_{\mathrm{r}}, \chi\right) G_{2} \frac{\partial q_{2}\left(x, x_{\mathrm{r}}, \chi\right)}{\partial \nu}-B \Lambda_{1} \tilde{W} \Gamma_{2}^{-1} \dot{W}^{\mathrm{T}}\right) .
\end{aligned}
$$

Finally, the update laws (38) and (39) provide the following bound for the Lyapunov derivative:

$$
\begin{aligned}
& \dot{V}\left(x, x_{\mathrm{r}}, \chi, \tilde{\Theta}, \tilde{W}, \hat{r}\right) \leq-\frac{\sigma_{1}}{2} \operatorname{tr}\left(B \Lambda_{1} \tilde{\Theta} \Gamma_{1}^{-1} \tilde{\Theta}^{\mathrm{T}}\right)+\frac{\sigma_{1}}{2} \operatorname{tr}\left(B \Lambda_{1} \Theta^{*} \Gamma_{1}^{-1} \Theta^{* \mathrm{~T}}\right) \\
&-\frac{\sigma_{2}}{2} \operatorname{tr}\left(B \Lambda_{1} \tilde{W} \Gamma_{2}^{-1} \tilde{W}^{\mathrm{T}}\right)+\frac{\sigma_{2}}{2} \operatorname{tr}\left(B \Lambda_{1} W^{*} \Gamma_{2}^{-1} W^{* \mathrm{~T}}\right) \\
&-\left\|G_{2}^{\frac{1}{2}} e_{2}\left(x, x_{\mathrm{r}}, \chi\right)\right\|\left(\left\|G_{2}^{\frac{1}{2}} e_{2}\left(x, x_{\mathrm{r}}, \chi\right)\right\|-\left\|G_{2}^{\frac{1}{2}} \frac{\partial q_{2}\left(x, x_{\mathrm{r}}, \chi\right)}{\partial \nu} B \Lambda_{1}\right\| \varepsilon^{*}\right) .
\end{aligned}
$$

It follows from Theorem 1 that the solutions of (38), (39), (45), and (46) are ultimately bounded with convergence to the compact set $M_{\mathrm{c}}$, defined in (40).

\section{Applications}

Application of the NN-MRAC algorithm will be presented in this section for two different marine vehicles. First, the algorithm is applied to a fully actuated ASV, then to a nonminimum phase AUV. Both models have the same general form as presented in Section 2, and their differences will be pointed out. The theorems from the previous section will be applied to each case to explicitly define the control command and show the stability of the error dynamics. Numerical simulation results will be presented for each case.

\subsection{ASV}

An example of how to compute the hydrodynamic terms that appear in (9) for an ASV is provided in [19]. Since the modeling trends and dynamical behavior are similar for different surface vessels, using the model developed in [19] helps to quantify the dynamics and performance capabilities of surface vessels and facilitates the testing of the controller.

We assume that the vessel is equipped with two motors in the rear that can be rotated independently. This provides a control input of

$$
\tau \triangleq[X, Y, N]^{\mathrm{T}}
$$

consisting of two forces $X, Y \in \mathbb{R}$ along the $x_{\mathrm{b}}$ and $y_{\mathrm{b}}$ axes, respectively, as well as a yawing moment $N \in \mathbb{R}$. This results in a fully actuated vehicle for motion along the horizontal plane. Hence, $\hat{B}=B=M^{-1}$, which is positive definite because of the nature of the mass matrix. 


\section{Control Command}

The position tracking error is defined as $e_{1}\left(\eta, x_{1 \mathrm{r}}\right) \triangleq \eta-x_{1 \mathrm{r}}$. The corresponding error dynamics are given by

$$
\dot{e}_{1}\left(x(t), x_{2 \mathrm{r}}(t)\right)=\dot{\eta}(t)-\dot{x}_{1 \mathrm{r}}(t)=J(\eta(t)) \nu(t)-x_{2 \mathrm{r}}(t), \quad t \geq 0 .
$$

Following the notation and procedure introduced in Section 4 , let $\chi(t) \equiv 0$ and

$$
\begin{aligned}
Q_{1}\left(\eta, x_{1 \mathrm{r}}\right) & =I_{3}, \\
q_{2}\left(x, x_{\mathrm{r}}\right) & =J(\eta) \nu-x_{2 \mathrm{r}} .
\end{aligned}
$$

The quadratic Lyapunov function candidate

$$
V_{s}\left(e_{1}\right)=\frac{1}{2} e_{1}^{\mathrm{T}} P e_{1}
$$

is chosen, where $P>0$. Hence, (27) and (28) with (52), (53), and $\alpha\left(\eta, x_{1 \mathrm{r}}\right)=1$, provide

$$
e_{2}\left(x, x_{\mathrm{r}}\right)=J(\eta) \nu-x_{2 \mathrm{r}}+G_{1} P\left(\eta-x_{1 \mathrm{r}}\right),
$$

and the new Lyapunov function candidate becomes

$$
V^{*}\left(e_{1}, e_{2}\right)=\frac{1}{2} e_{1}^{\mathrm{T}} P e_{1}+\frac{1}{2} e_{2}^{\mathrm{T}} G_{2} e_{2} .
$$

Let $\Lambda_{1}=\Lambda_{2}=I_{3}$, so that the control command (23) is given by

$$
\tau^{*}\left(x, x_{r}, \hat{r}\right)=-\left[\Theta^{*} w\left(x, x_{r}, \hat{r}\right)+\delta^{*}(x)\right],
$$

where the parameters defined in (24), (25), and (26) become

$$
\begin{aligned}
\Theta^{*}= & M \\
w\left(x, x_{r}, \hat{r}\right)= & J^{\mathrm{T}}(\eta)\left(\frac{\partial q_{2}\left(x, x_{\mathrm{r}}\right)}{\partial \eta} J(\eta) \nu-\dot{x}_{2 \mathrm{r}}+G_{1} P\left(J(\eta) \nu-x_{2 \mathrm{r}}\right)+e_{2}\left(x, x_{\mathrm{r}}\right)\right. \\
& \left.+G_{2}^{-1} e_{1}^{\mathrm{T}}\left(\eta, x_{1 \mathrm{r}}\right) P\right) \\
\delta^{*}(x)= & M f(x) .
\end{aligned}
$$

With this control command, we find the derivative of (56) to be

$$
\dot{V}^{*}\left(e_{1}, e_{2}\right)=-e_{1}^{\mathrm{T}} G_{1} e_{1}-e_{2}^{\mathrm{T}} G_{2} e_{2},
$$

which is negative definite, and the tracking error converges to zero in accordance with Theorem 2 .

Introducing the parameter estimates, the above control command is modified according to (37) in Theorem 3: 


$$
\tau(t)=-\left[\Theta(t) w\left(x(t), x_{\mathrm{r}}(t), \hat{r}(t)\right)+W(t) \sigma(x(t))\right], \quad t \geq 0 .
$$

The parameter estimates (38) and (39) become

$$
\begin{aligned}
\dot{\Theta} & =J^{\mathrm{T}}(\eta) G_{2}^{\mathrm{T}} e_{2}\left(x, x_{\mathrm{r}}\right) w^{\mathrm{T}}\left(x, x_{\mathrm{r}}, \hat{r}\right) \Gamma_{1}-\sigma_{1} \Theta, \\
\dot{W} & =J^{\mathrm{T}}(\eta) G_{2}^{\mathrm{T}} e_{2}\left(x, x_{\mathrm{r}}\right) \sigma^{\mathrm{T}}(x) \Gamma_{2}-\sigma_{2} W,
\end{aligned}
$$

which, according to Theorem 3, guarantees that the dynamics of the error and parameter estimates are ultimately bounded.

\section{Numerical Simulations}

In this section maneuvers will be performed to test the controller performance capabilities on the ASV.

The reference system, as described previously, consists of three uncoupled second-order differential equations. The constants describing the reference system dynamics are $\omega_{0}=0.2 I_{3}$ and $\zeta=\operatorname{diag}(0.7,0.7,0.45)$. Other constants chosen for the controller include $\Gamma_{1}=10 I_{3}, \Gamma_{2}=10 I_{12}, G_{1}=G_{2}=I_{3}$, $\sigma_{1}=\sigma_{2}=0.01$, and $P=I_{3}$.

\section{Circular Trajectory Results}

The first maneuver performed on the ASV is a circular trajectory about the origin of the EFF, defined by

$$
\begin{aligned}
x_{\mathrm{d}} & =A \sin (\omega t+\phi), \\
y_{\mathrm{d}} & =A \cos (\omega t+\phi), \\
\psi_{\mathrm{d}} & =-(\omega t+\phi), \quad t \geq 0 .
\end{aligned}
$$

The simulation was performed for a radius $A=10 \mathrm{~m}$, an angular velocity $\omega=\frac{2 \pi}{75} \mathrm{rad} / \mathrm{s}$, and a phase angle of $\phi=\frac{\pi}{4}$. The behavior of the vehicle is simulated for $75 \mathrm{~s}$, or one complete cycle. The corresponding trajectory is shown in Figure 1. The vehicle converges quickly to the desired trajectory while staying relatively close to the reference system. However, undesirable fluctuations in the control input can be seen for the first $10 \mathrm{~s}$ of the simulation. These are caused by the coupling between sway and yaw, which the reference system fails to account for.

\section{Octomorphic Trajectory Results}

The octomorphic trajectory is given by the following parametric equations:

$$
\begin{aligned}
& x_{\mathrm{d}}(t)=2 A \sin \left(\frac{\omega t}{2}\right), \\
& y_{\mathrm{d}}(t)=A \sin (\omega t), \quad t \geq 0 .
\end{aligned}
$$



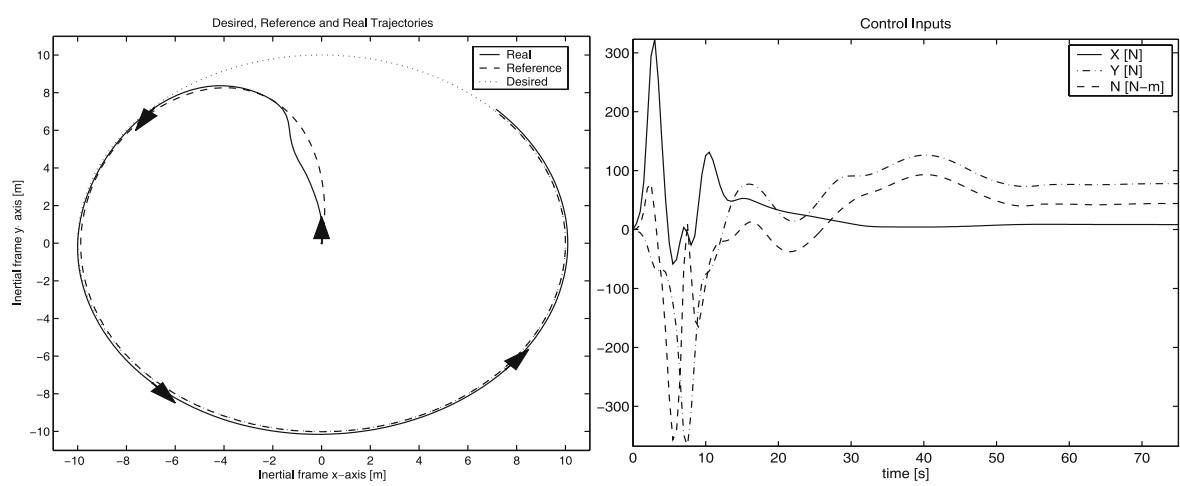

Fig. 1. Circular trajectory and corresponding control command, $\tau(t)$.

The tangential angle for this curve, corresponding to the desired orientation, is given by

$$
\psi_{\mathrm{d}}(t)=\tan ^{-1}\left(\frac{\dot{y}_{\mathrm{d}}(t)}{\dot{x}_{\mathrm{d}}(t)}\right)=\tan ^{-1}\left(\frac{\cos (\omega t)}{\cos \left(\frac{\omega t}{2}\right)}\right), \quad t \geq 0 .
$$

This maneuver is more complex than the circular trajectory that was tracked in the previous section. Tracking a circular trajectory involves a single turn with a constant turning radius. The octomorphic trajectory involves both left and right turns as well as straight lines.

Once again undesirable fluctuations in the control input are observed (see Figure 2), caused by the coupling between sway and yaw, which the reference system fails to capture.
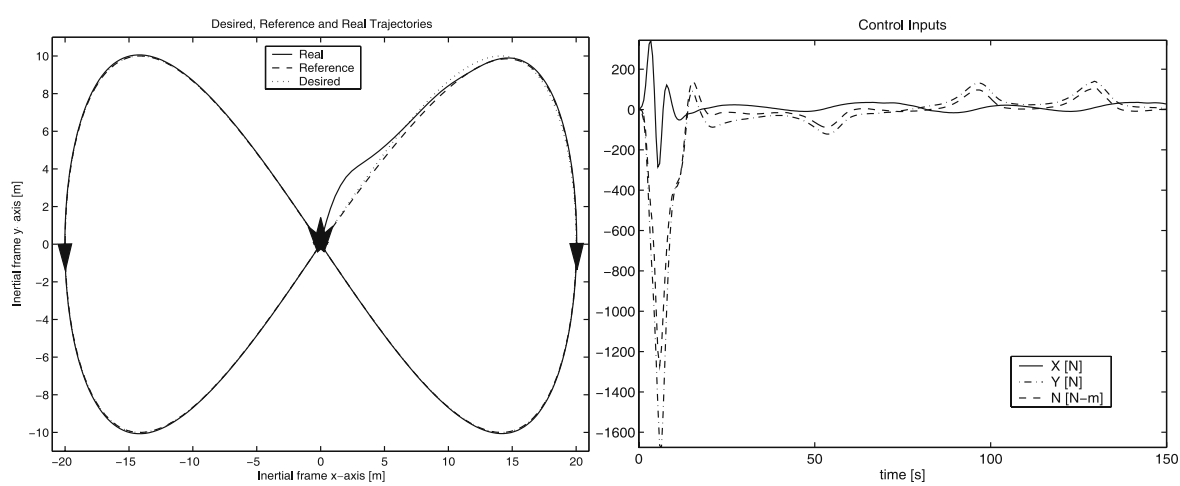

Fig. 2. Octomorphic trajectory and corresponding control command, $\tau(t)$. 


\subsection{AUV}

The control algorithm will now be applied to the most commonly used propulsion system available on AUVs as well as surface vessels: a thruster, used for propulsion, and a rudder for steering, or, equivalently, a vectored thruster $([14,15])$. The propulsion system considered provides two independent control commands, while the vehicle has three degrees of freedom. The vehicle will thus have fewer independent actuators than degrees of freedom, making it underactuated. The corresponding dynamic model is characterized by unstable zero dynamics, as mentioned in $[7,14,15]$, and the system is said to be nonminimum phase. Furthermore, $m=2$ and the available control command $\tau \in \mathbb{R}^{2}$ appearing in (9) is of the form

$$
\tau \triangleq\left[\tau_{1} \tau_{2}\right]^{\mathrm{T}}
$$

where $\tau_{1}$ and $\tau_{2}$ correspond to the surge and the sway force, respectively.

\section{Tracking Errors}

The error in position in the EFF and BFF are respectively defined by

$$
\begin{aligned}
e_{\mathrm{p}}\left(x_{1 \mathrm{r}}, \eta_{\mathrm{s}}\right) & \triangleq \eta_{\mathrm{s}}-x_{1 \mathrm{r}}, \\
\tilde{e}\left(x_{1 \mathrm{r}}, \eta\right) & \triangleq J_{\mathrm{s}}^{-1}(\psi) e_{\mathrm{p}}\left(x_{1 \mathrm{r}}, \eta_{\mathrm{s}}\right)=\left[\tilde{e}_{1}\left(x_{1 \mathrm{r}}, \eta_{\mathrm{s}}\right) \tilde{e}_{2}\left(x_{1 \mathrm{r}}, \eta_{\mathrm{s}}\right)\right]^{\mathrm{T}},
\end{aligned}
$$

where $e_{\mathrm{p}}\left(x_{1 \mathrm{r}}, \eta_{\mathrm{s}}\right)$ is the error in the EFF, $\tilde{e}\left(x_{1 \mathrm{r}}, \eta\right)$ is this same error in position, but projected in the BFF, $\eta_{\mathrm{s}} \triangleq\left[x_{\mathrm{N}} y_{\mathrm{E}}\right]^{\mathrm{T}}$ is the actual position of the vehicle, $x_{1 \mathrm{r}} \in \mathbb{R}^{2}$ is the position of the reference system that the vehicle is tracking, and

$$
J_{\mathrm{s}}(\psi) \triangleq\left[\begin{array}{cc}
\cos \psi & -\sin \psi \\
\sin \psi & \cos \psi
\end{array}\right] .
$$

Using (7) and (67), we obtain the following first and second time derivatives for $\tilde{e}(t)$ :

$$
\begin{aligned}
\dot{\tilde{e}}\left(x, x_{r}\right) & =\nu_{\mathrm{s}}-J_{\mathrm{s}}^{-1}(\psi) \dot{x}_{1 \mathrm{r}}+r S \tilde{e} \\
\ddot{\tilde{e}}\left(x, x_{r}, \tau, \hat{r}\right) & =\dot{\nu}_{\mathrm{s}}(x, \tau)-J_{\mathrm{s}}^{-1}(\psi)\left(\dot{x}_{2 \mathrm{r}}+r S \dot{\eta}_{\mathrm{r}}\right)+\dot{r} S \tilde{e}\left(x_{1 \mathrm{r}}, \eta\right)+r S \dot{\tilde{e}}\left(x, x_{r}\right),
\end{aligned}
$$

where $\nu_{\mathrm{s}} \triangleq[u v]^{\mathrm{T}}$, and $S$ is the following skew-symmetric matrix,

$$
S \triangleq\left[\begin{array}{cc}
0 & 1 \\
-1 & 0
\end{array}\right]
$$

Note that the control action $\tau(t)$ appears explicitly in the expression of $\ddot{\tilde{e}}(t)$ (70) through $\dot{\nu}_{\mathrm{s}}(t)$. The distance between the vehicle and its desired position is defined as 


$$
e_{\mathrm{d}}\left(x_{1 \mathrm{r}}, \eta_{\mathrm{s}}\right) \triangleq\left\|\tilde{e}\left(x_{1 \mathrm{r}}, \eta_{\mathrm{s}}\right)\right\|=\left\|e_{\mathrm{p}}\left(x_{1 \mathrm{r}}, \eta_{\mathrm{s}}\right)\right\|
$$

The time derivative of such a distance is given by

$$
\dot{e}_{\mathrm{d}}\left(x_{\mathrm{r}}, x\right)=\frac{1}{e_{\mathrm{d}}\left(x_{1 \mathrm{r}}, \eta_{\mathrm{s}}\right)} \tilde{e}^{\mathrm{T}}\left(x_{1 \mathrm{r}}, \eta_{\mathrm{s}}\right) \dot{\tilde{e}}\left(x_{1 \mathrm{r}}, \eta_{\mathrm{s}}\right) .
$$

Next, we define the error $\beta\left(x_{1 \mathrm{r}}, \eta_{\mathrm{s}}\right) \in(-\pi, \pi]$, which is the angle between the longitudinal axis of the vehicle and the direction of the desired position [1]. It can be computed as follows:

$$
\beta\left(x_{1 \mathrm{r}}, \eta_{\mathrm{s}}\right) \triangleq \tan ^{-1}\left(\frac{\tilde{e}_{2}\left(x_{1 \mathrm{r}}, \eta_{\mathrm{s}}\right)}{\tilde{e}_{1}\left(x_{1 \mathrm{r}}, \eta_{\mathrm{s}}\right)}\right),
$$

and its time derivative is given by

$$
\dot{\beta}\left(x_{\mathrm{r}}, x\right)=\frac{1}{e_{\mathrm{d}}^{2}\left(x_{1 \mathrm{r}}, \eta_{\mathrm{s}}\right)} \tilde{e}^{\mathrm{T}}\left(x_{1 \mathrm{r}}, \eta_{\mathrm{s}}\right) S \dot{\tilde{e}}\left(x_{1 \mathrm{r}}, \eta_{\mathrm{s}}\right) .
$$

We will consider the tracking error $e_{1}\left(x_{1 \mathrm{r}}, \eta_{\mathrm{s}}\right)=\left[e_{\mathrm{d}}\left(x_{1 \mathrm{r}}, \eta_{\mathrm{s}}\right) \beta\left(x_{1 \mathrm{r}}, \eta_{\mathrm{s}}\right)\right]^{\mathrm{T}}$. As $\beta\left(x_{1 \mathrm{r}}, \eta_{\mathrm{s}}\right)$ converges to zero, the vehicle will orient itself toward its desired position.

We can note that $\beta\left(x_{1 \mathrm{r}}, \eta_{\mathrm{s}}\right)$ is not defined for $e_{\mathrm{d}}\left(x_{1 \mathrm{r}}, \eta_{\mathrm{s}}\right)=0$, which implies that we can not guarantee that $e_{1}\left(x_{1 \mathrm{r}}, \eta_{\mathrm{s}}\right)=0$ if and only if $\eta_{\mathrm{s}}=x_{1 \mathrm{r}}$. We are, however, still able to apply Theorem 2 , but we will prove ultimate boundedness of the error dynamics rather than asymptotic stability. In particular, keeping in mind that perfect tracking is not achievable in the case of a nonminimum phase system [16], we will control the vehicle in such a fashion that $e_{1}\left(x_{1 \mathrm{r}}, \eta_{\mathrm{s}}\right)$ converges toward a compact set.

\section{Control Command}

The time derivative of $e_{1}\left(x_{1 \mathrm{r}}, \eta_{\mathrm{s}}\right)$ is given by $(22)$, with

$$
\begin{aligned}
& Q_{1}\left(x_{1 \mathrm{r}}, \eta_{\mathrm{s}}\right)=\left[\begin{array}{cc}
1 & 0 \\
0 & \frac{1}{e_{\mathrm{d}}}
\end{array}\right] J_{s}^{-1}(\beta), \\
& q_{2}\left(x, x_{\mathrm{r}}, \chi\right)=\nu_{\mathrm{s}}-J_{\mathrm{s}}^{-1}(\psi) \dot{x}_{1 \mathrm{r}}+\chi,
\end{aligned}
$$

where $\chi$ is a known exogenous signal whose time derivative is given by

$$
T \dot{\chi}\left(x_{1 \mathrm{r}}, \eta, \chi\right)=r S \tilde{e}\left(x_{1 \mathrm{r}}, \eta_{\mathrm{s}}\right)-\chi,
$$

where $T$ is a diagonal matrix with positive constant elements. We note that $\operatorname{det}\left[\mathrm{Q}_{1}\left(\mathrm{x}_{1 \mathrm{r}}, \eta_{\mathrm{s}}\right)\right] \neq 0$. We now consider the following Lyapunov function candidate $V_{s}\left(e_{1}\right)$ :

$$
V_{s}\left(e_{1}\right)=e_{\mathrm{d}} \sin ^{2}\left(\frac{\beta}{2}\right)+\frac{1}{2} e_{\mathrm{d}}^{2}
$$


Note that

$$
\frac{\mathrm{d} V_{s}\left(e_{1}\right)}{\mathrm{d} e_{1}}=\left[\sin ^{2}\left(\frac{\beta}{2}\right)+e_{\mathrm{d}} \quad \frac{1}{2} e_{\mathrm{d}} \sin (\beta)\right],
$$

and that $\frac{\mathrm{d} V_{s}\left(e_{1}\right)}{\mathrm{d} e_{1}}=0$ if and only if $e_{1}=0$.

The velocity error given by (28) is of the form

$$
e_{2}\left(x, x_{\mathrm{r}}, \chi\right)=\nu_{\mathrm{s}}-J_{\mathrm{s}}^{-1}(\psi) \dot{x}_{1 \mathrm{r}}+\chi+e_{\mathrm{d}} G_{1} J_{s}(\beta)\left[\begin{array}{c}
\sin ^{2}\left(\frac{\beta}{2}\right)+e_{\mathrm{d}}-a \\
\frac{1}{2} e_{\mathrm{d}} \sin (\beta)
\end{array}\right],
$$

where the dependencies of $\beta$ and $e_{\mathrm{d}}$ from $x_{\mathrm{r}}$ and $x$ were omitted, $a>0$ is an arbitrary constant that will measure the maximum allowable tracking error. As mentioned in [16], perfect tracking for a nonminimum phase system is not achievable. Accordingly, the control design objective for such a system should not be perfect tracking, but bounded-error tracking. Therefore, the expression of $e_{2}$ given by (28) is altered by introducing the $-a$ term. This introduces a position error corresponding to the distance between the vehicle and its desired position, which avoids unstable vehicle behavior.

We now consider the new Lyapunov function candidate

$$
V^{*}\left(e_{1}, e_{2}\right)=V_{s}\left(e_{1}\right)+\frac{1}{2} e_{2}^{\mathrm{T}} G_{2} e_{2} .
$$

Using the control command (23) with

$$
\begin{aligned}
\Theta^{*}= & \left(B \Lambda_{1}\right)^{-1} \Lambda_{2}, \\
w\left(x, x_{\mathrm{r}}, \chi, \hat{r}\right)= & \frac{\partial q_{2}\left(x, x_{\mathrm{r}}, \chi\right)}{\partial \eta} J(\eta) \nu-J_{\mathrm{s}}^{-1}(\psi) \dot{x}_{\mathrm{r}}+T^{-1}\left(r S \tilde{e}\left(x_{1 \mathrm{r}}, \eta_{\mathrm{s}}\right)-\chi\right) \\
& -\dot{q}_{2 \operatorname{des}}\left(x, x_{\mathrm{r}}, \chi\right)+e_{2}\left(x, x_{\mathrm{r}}, \chi\right)+G_{2}^{-1} J_{s}(\beta)\left[\begin{array}{c}
\sin ^{2}\left(\frac{\beta}{2}\right)+e_{\mathrm{d}} \\
\frac{1}{2} e_{\mathrm{d}} \sin (\beta)
\end{array}\right],(83) \\
\delta^{*}= & \left(B \Lambda_{1}\right)^{-1} f(x),
\end{aligned}
$$

where

$$
\Lambda_{1} \triangleq\left[\begin{array}{lll}
1 & 0 & 0 \\
0 & 1 & 0
\end{array}\right], \quad \Lambda_{2} \triangleq\left[\begin{array}{ll}
1 & 0 \\
0 & 1 \\
0 & 0
\end{array}\right]
$$

we obtain the following time derivative for the above Lyapunov function candidate:

$$
\dot{V}^{*}\left(e_{1}, e_{2}\right)=-e_{2}^{\mathrm{T}} G_{2} e_{2}-e_{\mathrm{d}}\left[\sin ^{2}\left(\frac{\beta}{2}\right)+e_{\mathrm{d}} \frac{1}{2} \sin (\beta)\right] G_{1}\left[\begin{array}{c}
\sin ^{2}\left(\frac{\beta}{2}\right)+e_{\mathrm{d}}-a \\
\frac{1}{2} \sin (\beta)
\end{array}\right] .
$$

Recognizing that $V^{*}\left(e_{1}, e_{2}\right)>0$ and $\dot{V}^{*}\left(e_{1}(t), e_{2}(t)\right)<0, t \geq 0$, provided that $e_{\mathrm{d}} \geqslant a$, it follows from Theorem 1 that the error dynamics are ultimately bounded. 
Introducing the parameter estimates, the control command is modified according to (37) in Theorem 3,

$$
\tau(t)=-\Lambda_{1}\left[\Theta(t) w\left(x(t), x_{\mathrm{r}}(t), \hat{r}(t)\right)+W(t) \sigma(x(t))\right], \quad t \geq 0 .
$$

The parameter estimates (38) and (39) become

$$
\begin{aligned}
\dot{\Theta} & =\Lambda_{2} G_{2}^{\mathrm{T}} e_{2}\left(x, x_{\mathrm{r}}, \chi\right) w^{\mathrm{T}}\left(x, x_{\mathrm{r}}, \chi, \hat{r}\right) \Gamma_{1}-\sigma_{1} \Theta, \\
\dot{W} & =\Lambda_{2} G_{2}^{\mathrm{T}} e_{2}\left(x, x_{\mathrm{r}}, \chi\right) \sigma^{\mathrm{T}}(x) \Gamma_{2}-\sigma_{2} W .
\end{aligned}
$$

It follows that the dynamics of the error and parameter estimates are ultimately bounded, in accordance with Theorem 3.

\section{Circular Trajectory Simulation Results}

The first maneuver we will attempt is a counterclockwise circle of radius $10 \mathrm{~m}$ at a velocity of $1 \mathrm{~m} / \mathrm{s}$, with the following initial conditions:

$$
\eta(0)=\left[\begin{array}{lll}
0 & 0 & 0
\end{array}\right]^{\mathrm{T}}, \quad \nu(0)=\left[\begin{array}{lll}
0 & 0 & 0
\end{array}\right]^{\mathrm{T}} .
$$

The reference model initial conditions are

$$
x_{\mathrm{r} 1}(0)=\left[\begin{array}{lll}
0.4 & 0
\end{array}\right]^{\mathrm{T}}, \quad x_{\mathrm{r} 2}(0)=\left[\begin{array}{ll}
0 & 0
\end{array}\right]^{\mathrm{T}} .
$$

The natural frequency and damping matrices of the reference system are set at $0.2 I_{2}$ and $0.9 I_{2}$, respectively. The initial conditions chosen for the $\Theta$ and $W$ estimates are

$$
\Theta(0)=\left[\begin{array}{cc}
15 & 0 \\
0 & 50
\end{array}\right], \quad W(0)=\left[\begin{array}{cccccc}
-5 & 0 & -5 & 0 & 40 & 0 \\
0 & 10 & 0 & 10 & 0 & 10
\end{array}\right] .
$$

Furthermore, $\Gamma_{1}=I_{2}, \Gamma_{2}=I_{6}, a=0.4$, and $G_{1}=G_{2}=I_{2}$. The dynamic model of the vehicle corresponds to the Silent Quick Unmanned Intelligent Diver [14]. The values for $M, C(\nu)$, and $D(\nu)$ are given in [14]. Finally, the initial position of the desired trajectory is

$$
\eta_{\mathrm{ds}}(0)=[7.07117 .0711]^{\mathrm{T}} .
$$

As shown in Figure 3, the tracking performances are excellent.

\section{Octomorphic Trajectory Simulation Results}

For our second maneuver, we consider an octomorphic trajectory. The initial conditions are the same as for the previous example, except for the desired trajectory

$$
\eta_{\mathrm{d}}(0)=\left[\begin{array}{ll}
0 & 0
\end{array}\right]^{\mathrm{T}} .
$$

The result of this simulation are displayed in Figure 4. The tracking performances are very good. 

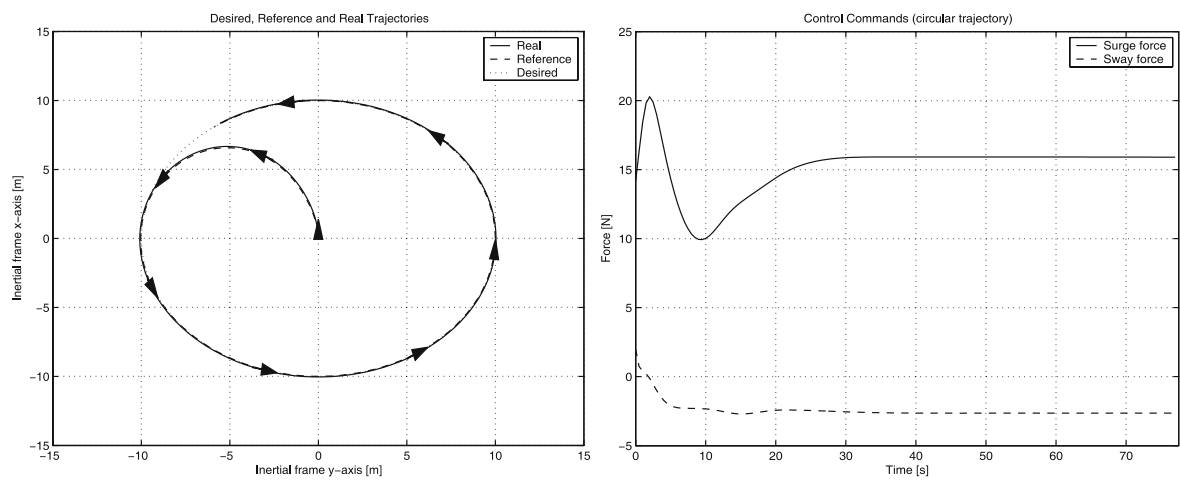

Fig. 3. Circular trajectory and corresponding control command, $\tau(t)$.
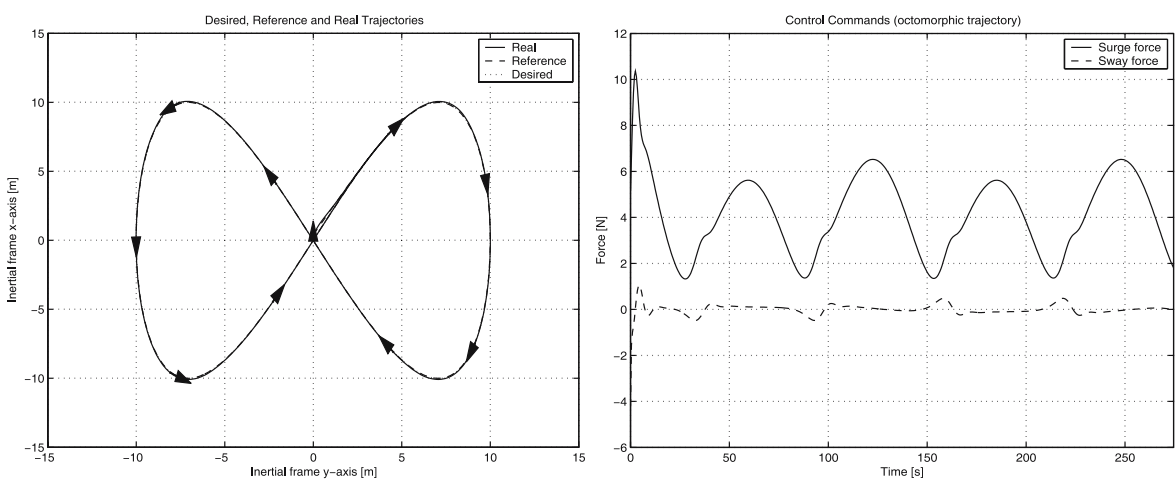

Fig. 4. Octomorphic trajectory and corresponding control command, $\tau(t)$.

\section{Conclusion}

A NN-MRAC algorithm was developed that uses Lyapunov stability theory to guarantee an ultimately bounded tracking error. The single layer neural network combines with the parameter update laws to eliminate the need to know any of the system dynamics, including its structure. This adds portability to the controller, which was demonstrated by its implementation on two different marine vehicles. Numerical simulations performed for an ASV showed excellent tracking performance despite a strong dependence on the reference system dynamics during the transient region. Equivalent results were seen for the AUV, where the complexity was heightened by the nonminimum phase properties of the system. 


\section{References}

1. Aguiar A, Pascoal A (2001) Regulation of a nonholonomic autonomous underwater vehicle with parametric modeling uncertainty using Lyapunov functions. 4178-4183. In: Proc. 40th IEEE Conf. Dec. Contr., Orlando, FL

2. Do K, Jiang Z.-P, Pan J (2002) Universal controllers for stabilization and tracking of underactuated ships. Sys. Contr. Lett., 47:299-317

3. Do K, Jiang Z.-P, Pan J (2003) Underactuated ship global tracking without measurement of velocities. In: Proc. 2003 IEEE Am. Contr. Conf., Denver, CO

4. Encarnacão PMM (2002) Nonlinear path following control systems for ocean vehicles. PhD Thesis, Instituto Superior Technico, Lisbon, Portugal

5. Fang Y, Zergeroglu E, de Queiroz M, Dawson D (2004) Global output feedback control of dynamically positioned surface vessels: an adaptive control approach. Mechatronics, 14(4):341-356

6. Fantoni I, Lozano R, Mazenc F, Pettersen K (2000) Stabilization of a nonlinear underactuated hovercraft. International Journal of Robust and Nonlinear Control, 10(8):645-654

7. Fossen T, Godhavn J.-M, Berge S, Lindegaard K.-P (1998) Nonlinear control of underactuated ships with forward speed compensation. 121-126., In: Proc. IFAC NOLCOS98, Enschede, Netherlands

8. Fossen TL (1999) Guidance and control of ocean vehicles. John Wiley \& Sons Ltd., Chichester, England

9. Godhavn J.-M (1996) Nonlinear control of underactuated surface vessels, 975980. In: Proc. 35th IEEE Conf. Dec. Contr. Kobe, Japan

10. Hayakawa T, Haddad WM, Hovakimyan N, Chellaboina V (June 2003) Neural network adaptive control for nonlinear nonnegative dynamical systems, 561566. In: Proc. Amer. Contr. Conf., Denver, CO

11. Krstić M, Kanellakopoullos I, Kokotović P (1995) Nonlinear and adaptive control design. John Wiley and Sons, New York, NY

12. LaSalle J, Lefschets S (1961) Stability by Liapunov's direct method with applications. Academic Press, Reading, MA

13. Lewis FL, Yesildirek A, Liu K (1999) Neural network control of robot manipulators and nonlinear systems. CRC Press, London, UK

14. Morel Y (2002) Design of an adaptive nonlinear controller for an autonomous underwater vehicle equipped with a vectored thruster. Master's thesis, Florida Atlantic University, Department of Ocean Engineering

15. Morel Y, Leonessa A (2003) Adaptive nonlinear tracking control of an underactuated nonminimum phase model of a marine vehicle using ultimate boundedness. In: Proc. 42th IEEE Conf. Dec. Contr., Maui, HI

16. Slotine J, Li W (1991) Applied nonlinear control. Prentice Hall, Englewood Cliffs, NJ

17. Toussaint G, Basar T, Bullo F (2000a) $H^{\infty}$-optimal tracking control techniques for nonlinear underactuated systems, 2078-2083. In: Proc. 39th IEEE Conf. Dec. Contr., Sydney, Australia

18. Toussaint G, Basar T, Bullo F (2000b) Tracking for nonlinear underactuated surface vessels with generalized forces, 355-360. In: Proc. of the IEEE International Conference on Control Applications, Anchorage, AK

19. VanZwieten TS (2003) Dynamic simulation and control of an autonomous surface vehicle. Master's thesis, Florida Atlantic University, Department of Ocean Engineering 